\title{
J. J. Thomson y la génesis del descubrimiento del electrón
}

\author{
José Manuel Sánchez Ron
}

Arbor CLVIII, 622 (Octubre), 137-171 pp.

Al mismo tiempo que se introduce la figura de J. J. Thomson, y cómo llegó al descubrimiento del electrón, entendido éste como partícula elemental, se estudian las aportaciones de otros científicos (como, entre otros, Faraday, Maxwell, Crookes, Helmholtz o Stoney) a la identificación de una carga eléctrica básica. Finalmente, se aborda la cuestión de la recepción del trabajo de Thomson.

Si de Isaac Newton se dijo que afortunado él porque vio lo que ya nadie más podría volver a ver por primera vez, de Joseph John Thomson (1856-1940) se puede decir otro tanto: fue él, más que ningún otro, quien comprobó experimentalmente la existencia de una partícula "elemental" universal; esto es, un constituyente aparentemente básico de toda la materia: el electrón.

\section{Años de formacion: Cambridge y el Mathematical Tripos}

Thomson (o JJ, como le llamaban muchos de sus colegas) fue un producto típico de la educación científica victoriana, en la que, como veremos enseguida, la Universidad de Cambridge, y dentro de ella, el denominado Mathematical Tripos, ocupaban un lugar prominente.

Thomson nació el 18 de diciembre de 1856, en Cheetham Hill, un suburbio de Manchester, en el seno de una familia modesta (su padre vendía libros antiguos). Inició sus estudios científicos, en 1871, en el 
Owens College de Manchester, en donde tuvo la fortuna de contar con maestros como Balfour Stewart y Osborne Reynolds, profesores de filosofia natural (física) e ingeniería, respectivamente, del college.

En el otoño de 1876, Thomson entró (con una beca) a formar parte del famoso Trinity College de Cambridge, al que permanecería asociado toda su vida, llegando a ser master de él.

Estudiar en Cambridge era entonces no sólo un medio de reivindicar, o-las menos de las veces-intentar acceder a un status social elevado, para aquellos con inclinaciones científicas, poseía un atractivo añadido: el Mathematical Tripos, el sistema de exámenes, en el que predominaban las preguntas matemáticas y de física teórica o matemática, vigente en Cambridge desde mediados del siglo XVIII. ${ }^{1}$

El Tripos estaba dividido en dos partes; la primera duraba cuatro días, después venía un intervalo de diez días, durante los cuales se corregían los ejercicios realizados; aquellos que obtenían mejores resultados pasaban a la segunda parte, de mayor dificultad, que duraba cinco días. El estudiante que obtenía al final la calificación más alta era denominado Senior Wrangler, el segundo Second Wrangler, y así sucesivamente. Llegar a ser Senior Wrangler constituía casi un honor nacional. Entre los científicos que obtuvieron este título figuran George Gabriel Stokes (1841), Arthur Cayley (1842), Peter Tait (1852), Edward Routh (1854), Joseph Larmor (1880) y Arthur Eddington (1904).

La exigencia del Tripos obligaba a prepararse de manera específica para él, y para ello existían personas, científicos destacados ellos mismos los mejores, que se dedicaban a este tipo de enseñanza (lo hacían de forma particular; esto es, eran pagados directamente por sus alumnos). Así, Stokes, William Thomson, Tait y Maxwell fueron discipulos de William Hopkins, el más famoso de los maestros que preparaban para el Mathematical Tripos. Su éxito fue tan grande que fue denominado "the senior Wrangler maker». El preparador de JJ fue Edward Routh, un maestro consumado en mecánica analítica.

Con la excepción de Michael Faraday y James Prescott Joule, físicos experimentales ambos, la mayoría de los principales físicos británicos que trabajaron entre, aproximadamente, 1820 y 1900, estudiaron en Cambridge y se examinaron del Tripos. Esto hace que en ocasiones se hable de «la escuela de Cambridge», a la que además de los wranglers ya citados pertenecen otros como Rayleigh, Lamb, el matemático y físico matemático Edmund Whittaker y Jeans.

De una manera más cuantitativa, se puede medir el impacto del Mathematical Tripos del hecho de que a lo largo de la segunda mitad del siglo XIX cerca de la mitad de las cátedras de física de las uni- 
versidades británicas fueron ocupadas por wranglers, esto es, por personas que habían obtenido un grado de primera clase en el Mathematical Tripos ${ }^{2}$.

JJ Thomson se examinó del Tripos en enero de 1880, obteniendo el segundo lugar (Second Wrangler), detrás de Joseph Larmor, que más tarde se convertiría en Lucasian professor de la Universidad de Cambridge, y que, asimismo, sería uno de los físicos que más impulsarían la teoría del electrón ${ }^{3}$. Para Thomson este resultado fue satisfactorio; así, en su autobiografia (Recollections and Reflections) escribio: "Quedé muy satisfecho con el resultado, ya que el puesto que obtuve fue tan alto como nunca esperé obtener" 4 .

$\mathrm{Al}$ margen de que no se puede considerar ninguna deshonra quedar detrás de Larmor - mucho mejor físico matemático, por otra parte, que Thomson (y la dimensión matemática del Tripos era muy grande)-, no fue este caso el único de un gran físico que qued6 Second Wrangler: así, y a pesar de su gran capacidad matemática y física, James Clerk Maxwell no consiguio el primer puesto en el Mathematical Tripos cuando, en 1854, pasó el examen; fue Second Wrangler, detrás precisamente de Routh, el coach de Thomson.

En tiempos de Maxwell, al igual que en los de Thomson, el título de Senior Wrangler no era el único perseguido en Cambridge, aunque sí, desde luego, el más prominente; también era apetecible lograr el premio Adams, que había sido instituido en 1848, como homenaje al astrónomo John Couch Adams (1819-1892) por haber previsto la existencia del planeta Neptuno. Se concedía cada dos años, al mejor trabajo sobre un tema de matemáticas, astronomía u otra rama de la filosofia natural (lo que en la actualidad entendemos por física) propuesto por un comité.

Maxwell se presentó al premio convocado en 1856 bajo el tema del "movimiento de los anillos de Saturno" (por entonces la cuestión de la naturaleza de los anillos permanecía abierta; podía tratarse de un sistema sólido o de uno líquido, o también de pequeños cuerpos muy próximos, aunque existía una cierta tendencia a favorecer la hipótesis de que era de naturaleza líquida), y en junio de 1857 supo que lo había ganado. Como era habitual, la memoria fue publicada -incorporando algunos detalles elaborados tras la concesión del premio-, bajo el título: On the Stability of the Motion of Saturn's Rings ${ }^{5}$.

JJ Thomson también pudo consolarse con la obtención del premio Adams, el correspondiente a 1882 , que había sido convocado para el tema de «Una investigación original de la acción entre dos vórtices cerrados en un fluido incompresible perfecto". La memoria de Thomson 
estuvo dedicada al movimiento de los anillos vorticiales, y fue publicada como libro bajo el título: $A$ Treatise on the Motion of Vortex Rings (Macmillan, Londres 1883).

\section{Primeras investigaciones}

El año después de graduarse, Thomson logró una fellowship del Trinity (lo normal era no intentar siquiera solicitar esta especie de beca hasta el segundo año, pero JJ fue ambicioso y tuvo éxito) ${ }^{6}$. Fue entonces cuando comenzo a investigar, desde el punto de vista teórico, en el campo de cargas eléctricas en movimiento, según la teoría electromagnética de Maxwell. De hecho, pronto publicó un artículo sobre el tema: "On the electric and magnetic effects produced by the motion of electrified bodies" ${ }^{7}$. He aquí como se refirio el propio Thomson a este trabajo en Recollections and Reflections (pp. 91-92):

"Después de mi elección para una Fellowship comencé algunas investigaciones matemáticas sobre cargas de electricidad en movimiento. Me ví atraído a esto por los bellos experimentos sobre rayos catódicos que habían sido realizados hacía poco por Crookes, y estaba ansioso de ver cual debía ser el comportamiento de partículas en movimiento en la teoría de Maxwell... Cargas de electricidad no figuraban en absoluto de manera prominente en esta teoría, y cuando Maxwell, al discutir la electrolisis tuvo a bien, como modo conveniente de expresion, hablar de la molécula de electricidad, dice la frase sin ninguna armonía con el resto de su tratado. Helmholtz, que era un gran defensor de la teoría, dice que no sabría explicar lo que era una carga eléctrica en la teoría de Maxwell, más allá del receptor de un símbolo".

Y en este punto, añadía: "Como la fuerza eléctrica cerca de una partícula electrificada varía muy rápidamente según se mueve la partícula, el comportamiento de una partícula cargada en movimiento debería proporcionar un método satisfactorio para comprobar la teoría. Desarrollé, por tanto, cual debería ser en esta teoría la fuerza magnética producida por la partícula en movimiento, y cual debería ser la fuerza mecánica sobre la partícula si actuase sobre ella un campo magnético externo".

Vemos en la cita precedente, cómo hacia 1881, dieciseis años antes de que escribiera el artículo que es considerado como el que marca el descubrimiento del electrón, JJ Thomson ya seleccionó problemas y desarrolló métodos de análisis teórico que le serían de gran utilidad 
- casi podríamos decir que imprescindibles- para realizar su mayor aportación a la física.

También aparecen en esa cita nombres propios y términos que constituyen elementos clave para comprender la génesis del descubrimiento del electrón, y que es preciso, por consiguiente, comentar: Maxwell, Crookes, Helmholtz, electrolisis, "carga eléctrica como un símbolo" y rayos catódicos. Y para comentarlos, hay que comenzar por retroceder hacia los tiempos de Maxwell.

\section{Faraday, Maxwell, electrolisis y "carga molecular"}

La progresiva consolidación de la teoría electromagnética de Maxwell no quiere decir que se poseyese una visión completa de los fenómenos electromagnéticos. Un ejemplo en este sentido es el de la corriente eléctrica. Entre las ideas que se manejaron se encontraba la de que el desplazamiento del éter electromagnético - que se suponía incompresible- era consecuencia (y seguía la dirección) de una acción lineal de la fuerza eléctrica, no siendo la corriente eléctrica a través de un conductor sino el subsiguiente flujo del éter a lo largo de él. El mecanismo de la formación de una carga electrostática en la superficie del conductor se representaba mediante un modelo en el que se introducía un líquido incompresible en un tubo, que estaba cerrado en sus extremos mediante una membrana flexible; el líquido empujaba la membrana hasta que las reacciones elásticas compensaban la presión del fluido. Así, cerca del final del tubo se producirían tensiones elásticas, que representarían las tensiones eléctricas, y que afectarían, por supuesto, también al exterior del tubo. Tal era una de las primeras representaciones mecánica, en este caso- del mecanismo de la corriente eléctrica, que, en palabras de Arthur Schuster, "pronto se convirtió en un artículo de dogma que retrasó el progreso" ${ }^{8}$.

Si se tratase únicamente del paso de la electricidad a través de conductores sólidos, entonces tal vez hubiera sido posible mantener representaciones tan simples como la anterior, pero ni la conducción a través de electrolitos, ni a través de gases pudo acomodarse a semejante esquema. Inicialmente, tanto líquidos como gases apenas fueron tomados en consideración, pero ésta era una situación que, inevitablemente, tenía que cambiar.

El punto de partida en tal dirección se encuentra en los estudios de Michael Faraday sobre la electrolisis, que demostraron que en el caso de conductores líquidos el paso de una cierta cantidad de elec- 
tricidad está asociado siempre con la transferencia de una cantidad definida de materia ${ }^{9}$. Este resultado condujo, como veremos enseguida, a la idea de que cada uno de los átomos o radicales que era liberado por la electrolisis transportaba una cantidad concreta de electricidad. Sin embargo, el propio Faraday no llegó tan lejos, ni siquiera, como señaló Pearce Williams, pensó en términos semejantes. A lo más que llegó fue a escribir lo siguiente ${ }^{10}$ : "si adoptamos la teoría o fraseología atómica, entonces los átomos de cuerpos que son equivalentes entre sí en su acción química ordinaria, tienen iguales cantidades de electricidad asociadas a ellos. Pero tengo que confesar que me molesta hablar de átomos; porque es muy fácil hablar de átomos, pero muy difícil formarse una idea clara de su naturaleza, especialmente cuando se están considerando cuerpos compuestos».

En cuanto a Maxwell, se dio cuenta de los problemas que originaba explicar las leyes de Faraday de la electrolisis si quería mantener sus propias ideas sobre la conducción. En este sentido, en el capítulo (el IV) dedicado a la electrolisis de su A Treatise on Electricity and Magnetism (1873) leemos ${ }^{11}$ :

«De todos los fenómenos eléctricos, la electrolisis aparece como el más adecuado para suministrarnos un entendimiento real de la verdadera naturaleza de la corriente eléctrica, porque encontramos corrientes de materia ordinaria y corrientes de electricidad que forman parte esencial del mismo fenómeno".

$\mathrm{Y}$, tras explicar la teoría de Clausius con detalle, proseguía (p. 379): "Pero si continuamos y suponemos que las moléculas de los iones dentro del electrolito están cargadas con una cierta cantidad definida de electricidad, positiva y negativa, de manera que la corriente electrolítica es simplemente una corriente de convección, encontramos que esta tentadora hipótesis nos conduce a un terreno muy difícil». No obstante, la dificultad no le impedía continuar explorando posibilidades ${ }^{11}$ :

"Supongamos, sin embargo, que pasamos sobre esta dificultad simplemente sosteniendo el hecho del valor constante de la carga molecular, y que llamamos a esta carga molecular, por conveniencia en la descripción, una molécula de electricidad.

Esta frase, imprecisa como es, y fuera de armonía con el resto de este tratado, nos permitirá al menos expresar claramente lo que se conoce acerca de la electricidad y apreciar las dificultades principales". 


\section{J.J. Thomson y la génesis del descubrimiento del electrón}

\section{Helmholtz y la existencia de una carga eléctrica «atómica"}

Mucho más precisas fueron las conclusiones que sobre la existencia -que no sobre su naturaleza- de la carga eléctrica extrajo del fenómeno electrolítico Hermann von Helmholtz (otro de los nombres mencionados por JJ en la cita utilizada con anterioridad) en la "Conferencia Faraday" que pronunció en la Chemical Society de Londres el 5 de abril de 1881.

Para lo que a nosotros nos interesa en la presente ocasión, es preciso destacar que para Helmholtz no era «en modo alguno necesario aceptar ninguna opinión concreta acerca de la naturaleza última del agente que denominamos electricidad. El propio Faraday evitó tanto como le fue posible efectuar manifestaciones afirmativas sobre este problema, aunque no ocultó su falta de inclinación a creer en la existencia de dos fluidos eléctricos opuestos» ${ }^{13}$. No obstante, en su discusión de los fenómenos electroquímicos, Helmholtz utilizó «el lenguaje de la vieja teoría dualista, que considera a la electricidad positiva y a la negativa como dos sustancias imponderables, porque tendremos que hablar sobre todo de relaciones de cantidad".

En cualquier caso, declaraba explícitamente que intentaría imitar a Faraday restringiéndose "cuidadosamente al dominio de los fenó. menos", lo que significaba que "no necesitaremos especular acerca de la naturaleza real de aquello que denominamos una cantidad de electricidad positiva o negativa. Llamándolas sustancias de signo opuesto, implicamos con este nombre nada más que el hecho de que una cantidad positiva nunca aparece o desaparece sin que una igual cantidad negativa aparezca o desaparezca al mismo tiempo en su entorno inmediato. A este respecto, se comportan realmente como si fuesen dos sustancias que no pueden ser generadas ni destruidas sino que pueden ser neutralizadas haciéndose imperceptibles mediante su unión".

Más adelante, y refiriéndose ya en concreto a las leyes de Faraday sobre la electrolisis, Helmholtz manifestaba ${ }^{14}$ :

\footnotetext{
«Establecida de esta manera, la ley de Faraday nos dice que a través de cada sección de un conductor tenemos siempre movimientos eléctricos y químicos equivalentes. La misma cantidad definida de electricidad positiva o negativa se mueve siempre con cada ión univalente, o con cada unidad de afinidad de un ión multivalente, y la acompaña durante todos sus movimientos por el interior del fluido electrolítico. A esta cantidad la podemos llamar la carga eléctrica del átomo".
} 
Más concretamente ${ }^{15}$ : «el resultado más notable de la ley de Faraday acaso sea este: si aceptamos la hipótesis de que las sustancias elementales están compuestas de átomos, no podemos evitar concluir que también la electricidad, positiva al igual que negativa, está dividida en partes definidas elementales, que se comportan como átomos de electricidad. Mientras se mueve en el líquido electrolítico, cada ión permanece unido con su equivalente o equivalentes eléctricos. En la superficie de los electrodos tiene lugar la descomposición si existe suficiente fuerza electromotriz, y entonces los iones emiten sus cargas eléctricas y se hacen neutros eléctricamente".

En otras palabras, en 1881, para Helmholtz, no se podía decir prácticamente nada acerca de los "átomos de electricidad"... salvo que existían.

\section{Stoney, la unidad de carga y el nombre «electrón»}

La «Conferencia Faraday» era, sin duda, una buena palestra pública, y las manifestaciones realizadas en 1881 por Helmholtz tuvieron bastante eco, más aún habida cuenta del prestigio que poseía el fisiólogo y físico alemán. No es extraño, por consiguiente, que muchos científicos adjudicasen a Helmholtz el mérito de haber sido el primero que propuso seriamente, con buenos argumentos, la existencia de una unidad de electricidad. Así, Hermann Ebert declaraba en un artículo sobre el calor de disociación, publicado en el número de septiembre de 1894 de Philosophical Magazine, que «en base a las leyes de la electrolisis de Faraday, von Helmholtz fue el primero en demostrar en el caso de electrolitos que se debe considerar que toda valencia está cargada con una cantidad mínima de electricidad, la "carga de valencia", que al igual que el átomo eléctrico ya no se puede dividir" ${ }^{16}$.

Tal vez se podría argumentar que la formulación de Ebert estaba suficientemente matizada como para dejar puertas abiertas a otras reclamaciones de prioridad, pero no lo consideró de forma semejante un físico irlandés: George Johnstone Stoney (1826-1911), fellow de la Royal Society y secretario de la Queen's University - precursora de la National University- de Irlanda entre 1857 y $1882^{17}$. Justo el mes siguiente a que apareciese el artículo de Ebert, Stoney publicaba una carta dirigida a los editores de la revista, titulada "Of the 'electron', or atom of electricity", en la que reclamaba sus derechos de prioridad ${ }^{18}$ : 
"Ahora bien, yo había señalado ya dos veces este importante hecho: primero, en la reunión de Belfast de la British Association for the Advancement of Science de agosto de 1874, en un artículo "On the physical units of Nature", en el que llamaba la atención sobre esta cantidad mínima de electricidad, como una de las tres [nota bene] unidades físicas, cuyas magnitudes absolutas nos suministra la Naturaleza, y que pueden formar la base de un cuerpo completo de unidades sistemáticas en las que no exista nada arbitrario. Leí de nuevo este mismo artículo ante la Royal Society de Dublín el 16 de febrero de 1881, y está publicado en las actas de aquella reunión, al igual que en el Phil. Mag. de mayo siguiente" ${ }^{19}$.

Y para reforzar su reclamación, comparaba unos párrafos de la conferencia de Helmholtz (precisamente los mismos que cité al final de la sección precedente: «el resultado más notable de la ley de Faraday...») con unos de su artículo de 1874/1881:

«Y, finalmente, la Naturaleza nos presenta, en el fenómeno de la electrolisis, una cantidad definida de electricidad que es independiente de los cuerpos concretos sobre los que actúa. Para que quede esto claro, expresaré la "Ley de Faraday" de la forma siguiente, que, como demostraré, la hará precisa: Para todo enlace químico que se rompe dentro de un electrolito, una cierta cantidad de electricidad, que es la misma en todos los casos, atraviesa el electrolito. A esta cantidad definida de electricidad la llamaré $\mathrm{E}_{1}$. Si hacemos de ella nuestra unidad de electricidad, habremos dado probablemente un paso importante en el estudio de los fenómenos moleculares".

En su carta a los editores del Philosophical Magazine, Stoney también recordaba el valor que había dado en 1874 para la carga electrónica elemental, $3 \times 10^{-11}$ en unidades C.G.S., y lo comparaba con el dado por otro autor citado por Ebert, el físico alemán Franz Richarz ${ }^{20}, 12,9 \times 10^{-11}$. El valor aceptado en la actualidad es $4,8 \times 10^{-10}$.

Como vemos, Stoney tenía realmente argumentos para reclamar, y no hay duda, por tanto, que su nombre debe ser citado en cualquier exposición que pretenda recuperar los orígenes del descubrimiento del electrón. Y no sólo por lo que he dicho hasta ahora, sino también porque fue él quien acuñó en 1891 el término «electrón" (antes, en $1874 / 1881$, había utilizado el término "the electromagnetic electrine") ${ }^{21}$. Una de las ocasiones - aparentemente, la primera- en que introdujo aquel año el nombre de «electrón" fue en la reunión anual de la British Association for the Advancement of Science, en donde afirmó ${ }^{22}$ : 
146

José Manuel Sánchez Ron

Figura 1

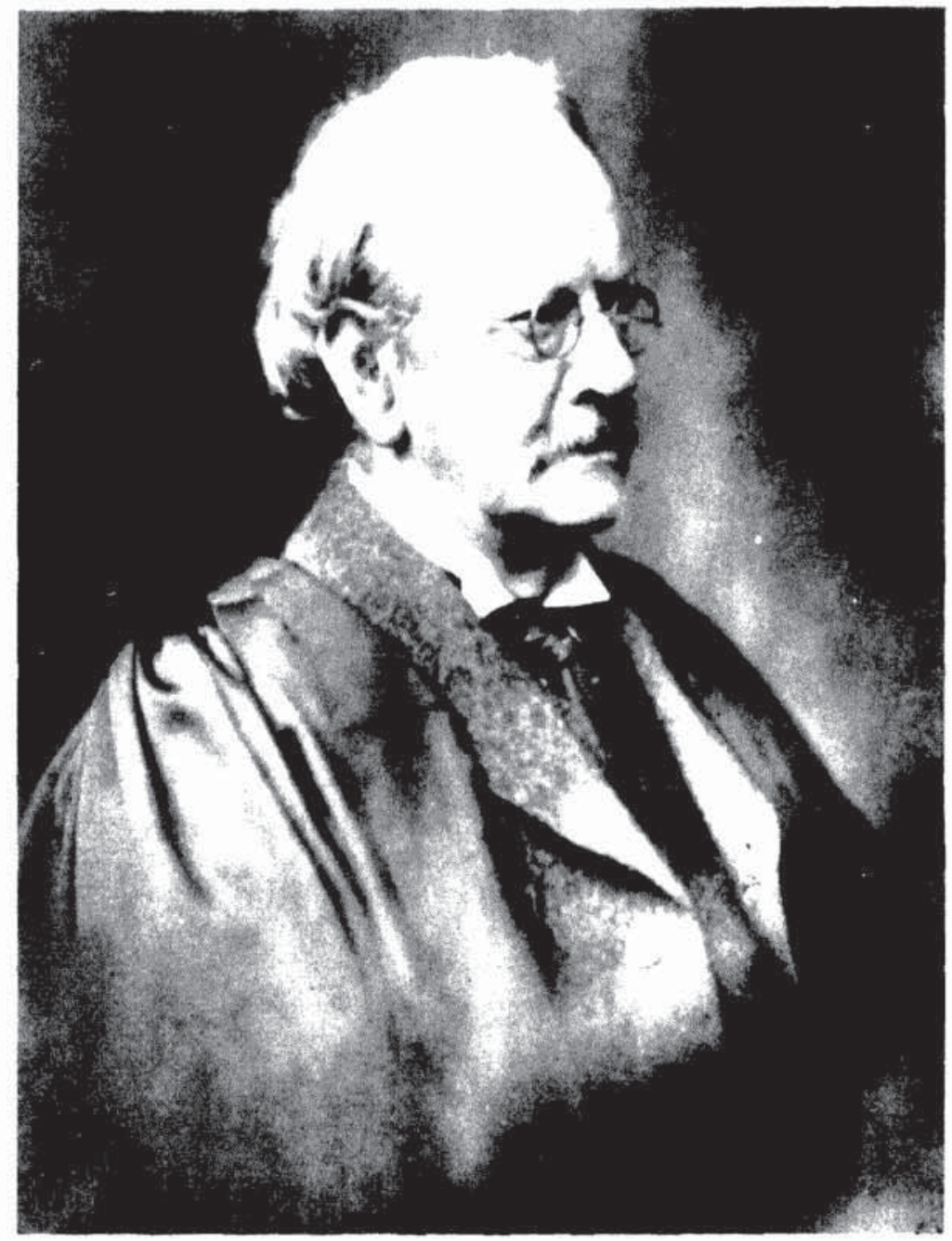

J. J. Thomson (1856-1940). Fotografia de la *W. F. Meggers Collection-. Cortesia de la Niels Bohr Library, American Institute of Physics. 


\section{J.J. Thomson y la génesis del descubrimiento del electrón}

"Las líneas del espectro de un gas se deben a algunos sucesos que tienen lugar dentro de las moléculas, y que son capaces de afectar al éter. Estos sucesos pueden ser descargas hertzianas entre moléculas que están electricizadas de manera diferente, o pueden ser debidos a los movimientos de esas inamovibles cargas eléctricas, cuya suposición ofrece la explicación más simple de la ley de electrolisis de Faraday... Diversas consideraciones... sugieren que la fuente de las líneas espectrales deben buscarse no en las descargas hertzianas, sino en el transportista de las cargas eléctricas fijas, que por conveniencia puede ser denominado el electrón".

\section{Parte de una historia mas compleja}

Naturalmente, la historia de las aportaciones, a propósito de la electrolisis, al establecimiento de la idea de una carga eléctrica elemental, no se agota con los científicos mencionados hasta el momento. Todo aquel que desee recuperar de forma más completa esa, sin duda fascinante, historia, debe prestar atención a nombres como, entre otros, el de Svante Arrhenius, cuya tesis doctoral (1883): Bestämning medelst polarisator av den galvaniska ledningsförmägan hos mycket utspädda vattenlösningar samt grunderna för en kemisk teori för elektrolyters lösning (Determinación de la conducción de soluciones acuosas altamente diluidas con la ayuda de un depolarizador y fundamentos para una teoría química de electrolitos en solución). La tesis de Arrhenius contiene contribuciones notables - y llevadas a cabo, probablemente, sin conocer los trabajos de Maxwell, Stoney o Helmholtz-, como la de que todos los iones se combinan con la misma cantidad de electricidad, negativa para el anión y positiva para el cation, lo que implicaba, sostenía, que la carga transportada por los iones se podía "atomizar" en cargas unitarias ${ }^{23}$.

\section{En el Laboratorio Cavendish}

A pesar de que aún no he completado el repaso de los nombres y conceptos mencionados por Thomson en su autobiografia (todavía falta por introducir a Crookes y, sobre todo, a los rayos catódicos), es hora ya de regresar a la biografía de JJ.

Aunque Thomson había comenzado su carrera de científico centrándose en estudios teóricos, no descuidó la investigación experimental, 
comenzando a realizar trabajos en este sentido en el Laboratorio Cavendish inmediatamente después de graduarse como Wrangler.

El Laboratorio Cavendish es todavía hoy uno de los centros de investigación científica más famosos de Cambridge ${ }^{24}$. Semejante fama se puede comprender fácilmente si se recuerda que entre sus directores figuran figuras como Maxwell, Rayleigh, JJ Thomson, Ernest Rutherford o William Bragg, sin olvidar que fue allí en donde se llevaron a cabo descubrimientos auténticamente fundamentales, como el de la estructura del ADN (debido a James Watson y Francis Crick).

El primer director del Cavendish fue Maxwell. En realidad, Maxwell fue designado (el 24 de febrero de 1871) para ocupar una cátedra recién creada, de Física Experimental ${ }^{25}$. Uno de los medios con que se dotó al catedrático fue la de un nuevo laboratorio. Este fue construido gracias a la generosidad de William Cavendish, séptimo duque de Devonshire, que había sucedido al Príncipe Consorte, el marido de la reina Victoria, en el puesto de Canciller de la Universidad de Cambridge. De hecho, durante algún tiempo después de ser nombrado catedrático, la tarea principal de Maxwell fue la de diseñar y supervisar la construcción del laboratorio (para ello, inspeccionó los laboratorios de física de William Thomson en Glasgow y de Robert Clifton en Oxford), que quedo terminado en 1873.

JJ comenzó a efectuar algunas visitas al Laboratorio cuando Maxwell era director. He aquí cómo se refirió a su entrada al Cavendish en su autobiografia ${ }^{26}$ :

"Comencé a trabajar en el Laboratorio Cavendish inmediatamente después de obtener mi título. No lo había hecho antes, aunque iba de vez en cuando al laboratorio para ver a Poynting o a Schuster, que estaban trabajando allí. No fui lo bastante afortunado como para ver a Maxwell en ninguna de estas ocasiones: estaba editando entonces los trabajos inéditos de Henry Cavendish y no se encontraba involucrado en ninguna investigación sistemática ${ }^{27}$. La idea de Maxwell de la función del laboratorio era que debería ser un lugar al que podrían ir aquellos que habían tomado el Mathematical Tripos y que, después de una breve preparación llevando a cabo mediciones precisas, realizasen alguna investigación original».

A la muerte de Maxwell, en 1879, John William Strutt, el tercer barón Rayleigh, fue elegido su sucesor, sin que sintiese demasiado entusiasmo; acepto, sobre todo debido a que su situación financiera no era entonces la mejor, así como para evitar ser criticado ${ }^{28}$. 


\section{J.J. Thomson y la génesis del descubrimiento del electrón}

Fue mientras Rayleigh era director cuando JJ Thomson comenzó realmente a trabajar en el Cavendish. Recurriendo de nuevo a Recollections and Reflections (p. 97):

«Comencé a trabajar en el laboratorio en 1880 intentando detectar la existencia de algunos efectos que yo pensaba que se deducían de la teoría de Maxwell: cambios en las fuerzas eléctricas en un dieléctrico producidos por fuerzas magnéticas. Los resultados que obtuve no fueron suficientemente conclusivos como para extraer de ellos conclusiones positivas y entonces me dediqué a una investigación sugerida por Lord Rayleigh. Se trataba de algunos efectos que se producían en el funcionamiento de anillos de inducción debido a la capacidad electrostática... Una descripción de esta investigación fue publicada en el Philosophical Magazine de julio de 1881. Empecé entonces, de nuevo a instancias de Lord Rayleigh, una investigación mucho más difícil y larga sobre la determinación del cociente entre las unidades electrostática y electromagnética de carga eléctrica que según la teoría de Maxwell debía ser igual a la velocidad de la luz; los trabajos de observadores anteriores habían demostrado que la diferencia no era grande".

Fue en aquella época cuando Thomson se presentó como candidato a la cátedra de Matemáticas Aplicadas de su viejo college, Owens College. Pero no tuvo éxito: el elegido resultó ser Arthur Schuster, que también había sido alumno allí y que además ya estaba dando clases en él (Oliver Lodge también fue candidato). En 1883, sin embargo, JJ fue elegido University lecturer en Cambridge, y cumo tal enseñó electrostática y electromagnetismo durante el curso académico 1883-84.

La carrera de Thomson iba, pues, progresando rápidamente. Buena muestra de ello es que la primavera de 1884 fue elegido miembro (fellow) de la Royal Society.

El escenario estaba dispuesto para el siguiente acto: en el otoño de 1884, Rayleigh anunció que dimitía de su puesto en el Cavendish. Una vez más se intentó que Kelvin aceptará el cargo, y de nuevo éste no aceptó. Hubo, por consiguiente, que anunciar libremente el puesto. Se presentaron cuatro candidatos, además de JJ Thomson: Osborne Reynolds, antiguo profesor de JJ en el Owens College, Arthur Schuster, que, como acabamos de ver, también había dado algunas clases en el Owens (asimismo, había trabajado como demostrator sin paga en el nuevo laboratorio de física) ${ }^{29}$, Richard T. Glazebrook, demostrator y lecturer en el propio Cavendish, y Joseph Larmor, que, como vimos, precedió a Thomson en el Tripos. Vista la lista, y el 
hecho de que Thomson no era particularmente conocido por sus trabajos experimentales, parecería que él era quien menos probabilidades tenía de ser elegido. No obstante, y aparentemente de forma sorprendente, fue él el seleccionado. Habida cuenta de cómo se desarrolló su carrera, hay que reconocer la sabiduría del comité que realizó la selección.

Como han señalado Edward Davis e Isobel Falconer, en su reciente y magnífica monografía dedicada a Thomson y el descubrimiento del electrón, es posible encontrar una cierta racionalidad en la decisión del comité que seleccionó a Thomson ${ }^{30}$. El programa tan práctico sobre unidades eléctricas que había favorecido Rayleigh durante su mandato en el Cavendish no era demasiado popular en Cambridge; se trataba de un programa excesivamente "práctico", palpablemente próximo a los valores «industriales» que la elitista comunidad universitaria consideraba secundarios —cuando no "vulgares" - ante la "verdadera cultura». Y tal sentimiento no debio ayudar a Glazebrook, que participaba completamente del espíritu de Rayleigh. JJ era, en este sentido, mucho mas aceptable, en tanto que sus investigaciones habían estado dirigidas al, para los dons de Cambridge, mucho más fundamental y apropiado problema de las relaciones entre éter y materia.

De los restantes candidatos, Osborne Reynolds era más un ingeniero que un físico, Schuster no era un Cambridge man, por mucho que hubiese trabajado durante algún tiempo en el Cavendish. En el caso de Larmor, Senior Wrangler en el Tripos, tal vez haya que señalar que estaba mucho más inclinado hacia las investigaciones teóricas que Thomson, quien, al menos, había manifestado el suficiente interés por la física experimental como para trabajar en el Cavendish.

\section{Rayos catódicos}

Con JJ ya instalado como professor en Cambridge, podemos regresar a la secuencia de desarrollos teóricos y experimentales de la que me había estado ocupando antes. Y lo primero que hay que señalar es que es dudoso que el fenómeno de la electrolisis hubiese sido, por sí solo, suficiente para conducir al descubrimiento del electrón ${ }^{31}$. Fueron, finalmente, los fenómenos producidos en las descargas eléctricas a través de gases enrarecidos los que llevarían al descubrimiento del electrón (para estos experimentos se utilizaban tubos en los que se hacía el vacío, y en cuyo interior se colocaban, además de un determinado gas, dos electrodos - el positivo, o ánodo, y el negativo, o cátodounidos a una batería). 


\section{J.J. Thomson y la génesis del descubrimiento del electrón}

El primer gran descubrimiento - el que conduciría finalmente al hallazgo del electrón-, asociado a los fenómenos producidos en tales descargas eléctricas fue el de los rayos catódicos, pero antes de referirme a ellos es necesario insistir en un punto, que aunque conocido en ocasiones pasa desapercibido, o no es valorado en su justa medida.

No hay ciencia sin experimentos (tampoco, por supuesto, sin teorías), pero éstos dependen en gran medida de los instrumentos que se poseen para «acceder" a los fenómenos que se producen en la naturaleza. Los rayos catódicos ejemplifican este hecho de manera magnífica, ya que sólo fueron descubiertos, durante la segunda mitad del siglo XIX, después del desarrollo de excelentes bombas de vacío, en particular las de Heinrich Geissler. Hasta entonces lo que se sabía era que descargas de alto voltaje a través de vacíos (no muy buenos) podían producir zonas luminosas. Pero con mejores vacíos se vio que tales «brillos" se iban debilitando progresivamente, y el cristal opuesto al polo negativo (el cátodo) comenzaba a brillar con una flourescencia.

Más concretamente, tenemos que durante sus investigaciones espectroscópicas, Julius Plücker, de Bonn, encontró (en 1858-59) que según se iba extrayendo el gas de uno de sus tubos la luminosidad que lo llenaba en un principio (producida por la diferencia de potencial existente entre los electrodos) disminuía progresivamente hasta que el cátodo aparecía rodeado por una delgada "envoltura" luminosa, de color variable según la naturaleza del gas introducido en el tubo, y separada del cátodo por un espacio oscuro, tanto más extenso cuanto mayor era el enrarecimiento de la atmósfera. Cuando la presión del gas llegaba a una millonésima de atmósfera, el espacio oscuro invadía todo el tubo, no observándose otra cosa que un pequeño círculo de luz violada en el extremo del cátodo, a la vez que el vidrio adquiría una intensa fosforescencia en la parte opuesta (para ello se utilizaron cátodos ligeramente cóncavos, con los que era posible dirigir tal fosforescencia hacia un punto en el otro extremo del tubo). Este fenómeno se denominó en principio emisión catódica; más tarde, cuando fue atribuído a la existencia, dentro del tubo, de radiaciones especiales emanadas directamente del cátodo, recibió el nombre de Kathodenstrahlen (rayos catódicos).

\section{Sobre la naturaleza —ondulatoria o corpuscular- de los rayos catódicos}

Hoy sabemos - gracias, especialmente, a JJ Thomson, lo veremos más adelante- que los rayos catódicos son corrientes de electrones. 
Proyectados desde el cátodo por repulsión electrica, navegan a través del espacio casi vacío del interior del tubo, golpean al cristal aportando energía a sus átomos, energía que se reemite entonces en forma de luz visible, siendo finalmente atraídos hacia el ánodo, a través del cual vuelven a la fuente de electricidad. Pero todo esto estaba lejos de la comprensión de los físicos cuando se descubrieron. Así, diferentes investigadores adoptaron distintas interpretaciones de este fenómeno. ¿Era, por ejemplo, la nueva radiación de naturaleza corpuscular u ondulatoria? Eugen Goldstein, físico del Observatorio de Postdam que publicó varios artículos sobre rayos catódicos, se inclinaba por la segunda opción, pero el británico William Crookes (1832-1919), uno de los científicos que más estudió la nueva radiación catódica, pensaba, por el contrario, que los rayos eran moléculas del gas encerrado en el tubo, que habían logrado adquirir una carga eléctrica negativa del cátodo, lo que hacía que éste las repeliese violentamente.

Por fin nos aparece el nombre de William Crookes, al que JJ Thomson se refirió, como ya vimos, cuando escribió que se vio "atraído a esto por los bellos experimentos sobre rayos catódicos que habían sido realizados hacía poco por Crookes». Me detendré un instante en él.

En 1876, William Crookes, dentro del contexto de sus investigaciones sobre los fenómenos que tenían lugar en el alto vacío, introdujo el concepto - ya utilizado por Faraday-de «materia radiante», el supuesto "cuarto estado de la materia" ${ }^{32}$. Dos años después comenzó una aparentemente nueva línea de investigación, dedicándose a estudiar los rayos catódicos, campo que hasta entonces había estado casi monopolizado por los alemanes ${ }^{33}$. Crookes creía que en la zona oscura existente en torno al cátodo cuando se producían descargas eléctricas en un tubo a baja presión, estaba relacionada, de alguna manera, con "la capa de presión molecular que produce movimientos en el radiómetro" ${ }^{34}$, un instrumento éste que él mismo diseñó y construyó en 1875: estaba formado por cuatro brazos, suspendidos de un saliente de acero, de manera que era capaz de girar horizontalmente; en el extremo de cada brazo llevaba un disco fino, ennegrecido en una cara, estando todas las caras negras orientadas hacia el mismo lado; todo este conjunto estaba encerrado en un tubo de vidrio herméticamente cerrado, en el que se había hecho el vacío más alto posible. El atractivo inmediato del radiómetro era que los brazos giraban libremente cuando se dirigía a él una luz intensa (por ello también se le llamaba "molino de luz»); aún se pueden comprar como objetos decorativos.

Esas líneas de presión molecular podían ser interpretadas como corrientes de materia radiante en un medio en el que existía un vacío 


\section{J.J. Thomson y la génesis del descubrimiento del electrón}

muy elevado, con lo que averigurar sus propiedades -0 , lo que era practicamente lo mismo, las de la radiación catódica- constituiría una forma excelente de avanzar en el conocimiento de la materia radiante. Y entre las propiedades que Crookes descubrió figuran algunas, dos sobre todo, que ayudaron a JJ Thomson en sus trabajos posteriores: (1) que las "corrientes de materia" eran desviadas por un imán, y (2) que se producía sombra cuando se interponían objetos opacos (como una cruz de Malta). En vista de tales resultados era natural pensar que la radiación catódica/materia radiante debía ser de naturaleza corpuscular.

Por otro lado, experimentos llevados a cabo por Heinrich Hertz en 1883 y 1891 parecían descartar la idea de que los rayos catódicos fuesen partículas cargadas eléctricamente; en su opinión, eran ondas de algún tipo ${ }^{35}$. Pero en 1895, Jean Perrin mejoró las técnicas de Hertz y demostró que estos rayos depositaban carga eléctrica negativa en un colector de carga introducido en el interior de un tubo de rayos catódicos ${ }^{36}$.

En general tal vez no sea exagerado afirmar que en Gran Bretaña predominaba la idea de que los rayos catódicos estaban formados por algún tipo de partículas o moléculas. Un buen ejemplo en este sentido se encuentra en la correspondencia que mantuvieron Stokes y Kelvin. Poco antes de que JJ Thomson comenzará a publicar sus seminales trabajos, el 10 de febrero de 1896, Stokes escribía a Kelvin ${ }^{37}$ :

«Estaba tan satisfecho con que las cosas parecidas a rayos que vienen del cátodo, y sobre las que actúa un imán, son corrientes de moléculas, o quizás átomos, que cuando me encontré accidentalmente con la expresión "Kathodostrahlen" [sic], supuse que se les llamaba rayos solamente en un sentido metafórico, como un término conveniente. Mencionando esto hace algún tiempo a [J.J.] Thomson, me dijo que eran varios los que los tomaban por rayos de algún tipo. Leyendo subsiguientemente algunos artículos alemanes, encontré que los autores sostenían fuertemente esta opinion, tanto que creo que miraban con cierto desdén a aquellos que pensaban de otra forma. No lo había hecho cuando hablé con Thomson, pero ahora he leido el artículo de Lenard ${ }^{38}$. Röntgen parece pensar que Lenard ha sentenciado la cuestión de que los Kathodostrahlen son rayos reales de alguna clase; aunque Röntgen distingue, lo que Lenard no hace, los rayos X de los Kathodostrahlen.

Me pregunto cual es su idea sobre la forma en que, en los experimentos de Lenard, pasan, o parecen pasar, los "Kathodostrahlen", a través de una ventana de aluminio, a un vacío casi tan perfecto como puede lograrlo. Mi idea es que es algo como la forma en que, en una fila de 
bolas de marfil en contacto, si la primera es golpeada la última sale despedida; no es que tome una noción tan groseramente meramente mecánica como esa. Para explicar los fenómenos ordinarios de la corriente molecular, se debe suponer que las rápidamente en movimiento moléculas (o átomos) están electrificadas. Mi idea es que en el caso común de una corriente (eléctrica) que fluye a lo largo de un cable algo tiene lugar en el cable similar a, si no de la misma naturaleza que, lo que tiene lugar en un electrolito cuando una corriente pasa a través de él; que existen movimientos de iones, o que responde a iones, en ambas direcciones; y lo mismo de nuevo en un tubo que contiene un gas rarificado; gas que si tiene la densidad adecuada mostrará la descarga estratificada".

\section{$\mathrm{Y}$ el 12 de febrero, Kelvin contestaba ${ }^{39}$ :}

"Con relación a la verdadera Kathodenstrom, es maravilloso cómo los alemanes la han llamado Kathodenstrahl, dejándose equivocar por Hittorf y Goldstein. Ellos, todos los que conozco excepto Helmholtz, han insistido con algo así como un fuerte espíritu partisano en un rayo de luz ondulatoria proveniente del cátodo y han rechazado ciegamente aceptar la conclusión de Varley de un torrente de moléculas, resultado corroborado por Crookes. Incluyo una copia marcada de mi conferencia de 1893 que puede mostrarle, o recordarle, algunas fechas y referencias sobre el tema" ${ }^{40}$.

El mismo año en que Stokes y Kelvin intercambiaban ideas sobre la naturaleza de los rayos catódicos, aunque unos meses después (en septiembre), el propio JJ Thomson, aprovechaba una parte de su conferencia inaugural de la sección A ("Mathematical and Physical Science») de la British Association for the Advancement of Science, reunida en Liverpool, para expresar sus opiniones. A pesar de que se repiten algunos datos ya mencionados (aunque de manera más sucinta), es interesante citar sus palabras ${ }^{41}$ :

«podemos, creo, dividir convenientemente los rayos que se producen en o cerca de un tubo de vacío atravesado por un campo magnético en tres clases, sin que ello implique que son necesariamente diferentes en carácter físico: Tenemos (1) los rayos catódicos dentro del tubo, que son desviados por un imán; (2) los rayos de Lenard fuera del tubo, que también son desviados por un imán; y (3) los rayos Röntgen, que no son, por lo que se sabe, desviados por un imán. Dos puntos de vista son los que se defienden a propósito de la naturaleza de los rayos catódicos; un punto de vista es que son partículas de gas que transportan cargas de electricidad negativa, y que se mueven con grandes velocidades, que han adquirido mientras viajaban a través del intenso campo eléctrico 


\section{J.J. Thomson y la génesis del descubrimiento del electrón}

que existe en los alrededores del electrodo negativo. La fosforescencia del cristal es según esta opinión producida por el impacto de estas partículas cargadas que se mueven rápidamente, aunque es una cuestión abierta si es producido por la violencia mecánica del impacto, o si es debido a un impulso electromagnético producido por un repentino cambio de la velocidad de la partícula cargada negativamente; esto es, no se sabe si es debido a causas mecánicas o eléctricas. Esa idea de la constitución de los rayos catódicos explica de manera sencilla la desviación de esos rayos en un campo magnético, y ha recibido recientemente una fuerte confirmación por los resultados de un experimento llevado a cabo por Perrin. Perrin colocó dentro del tubo en el que se había producido un vacío un recipiente cilíndrico metálico con un pequeño agujero en él, y conectó el cilindro con las hojas de un electroscopio de hojas de oro. Los rayos catódicos podían, por medio de un imán, ser guiados para pasar bien al cilindro a través de la abertura, o alejarse de él. Perrin encontró que cuando los rayos catódicos se introducían en el cilíndro las hojas de oro del electroscopio se separaban, y tenía una carga negativa, mostrando que el paquete de rayos catódicos encerrados en el cilíndro tenía una carga de electricidad negativa. Hace muchos años, Crookes había expuesto un disco conectado con un electroscopio de hojas de oro al bombardeo de rayos catódicos, y encontró que el disco recibía una carga ligeramente positiva; sin embargo, con semejante disposición las partículas cargadas tenían que ceder sus cargas al disco si las hojas de oro del electroscopio se veían afectadas, y sabemos que es extremadamente difícil, si no imposible, extraer la electricidad de un gas cargado meramente poniendo el gas en contacto con un metal... Es un rasgo del experimento de Perrin el que como actúa por inducción, las indicaciones del electroscopio son independientes de la comunicación de las cargas de electricidad del gas al cilindro, y como los rayos catódicos penetran en el interior del cilindro el electroscopio no se ve afectado, incluso si se produjese un efecto similar al que tiene lugar cuando luz ultravioleta incide sobre la superficie de un metal electronegativo que ha adquirido una carga positiva. Como semejante proceso no puede afectar a la cantidad total de electricidad dentro del cilindro, no afectará a las hojas de oro del electroscopio; de hecho, los experimentos de Perrin demuestran que los rayos catódicos transportan un carga de electricidad negativa".

$\mathrm{Y}$ así llegamos ya a las aportaciones de JJ Thomson al descubrimiento del electrón.

\section{J. J. Thomson y el descubrimiento del electrón}

Explicar de manera medianamente completa y sistemática la secuencia de investigaciones que llevó a JJ Thomson a descubrir el elec- 
trón, es algo que excede las posibilidades del presente artículo ${ }^{42}$. De lo expuesto hasta el momento, deberían haber quedado claros algunos de los motivos por los que JJ decidió abordar un problema - el de la naturaleza y estructura de los rayos catódicos- de gran actualidad e importancia. Sea como fuera, el hecho es que Thomson anunció que en su opinión los rayos catódicos eran "corpúsculos", pequeñas partículas cargadas negativamente, que formaban parte de los átomos, durante una de las "Friday evening lectures" - $C$ Conferencias nocturnas de los viernes" - de la Royal Institution: la que pronuncí el 30 de abril de 1897. Publicada en The Electrician, esta presentación precedió en casi seis meses la más detallada publicación del Philosophical Magazine (número de octubre) ${ }^{43}$.

En la Royal Institution, y tras llevar a cabo un repaso general de los trabajos que otros investigadores habían efectuado en el campo de los rayos catódicos, JJ anunciaba (p. 8) que había "realizado últimamente algunos experimentos que son interesantes por las implicaciones que tienen sobre las cargas transportadas por los rayos catódicos, al igual que acerca de la producción de rayos catódicos fuera del tubo" ${ }^{44}$. Una de esas implicaciones, la principal, era el valor que daba para el valor del cociente entre la masa y la carga, que Thomson daba al final de su charla ${ }^{45}$.

$$
m / e=1,6 \times 10^{-7} .
$$

Se trata de un valor, señalaba, "muy pequeño comparado con el valor $10^{-4}$ para el cociente entre la masa de un átomo de hidrógeno y la carga que transporta. Si no dispusiésemos más que de este resultado podríamos pensar que era probable que $e$ fuese mayor que la carga atómica del átomo, en lugar de que $m$ fuese menor que la masa del átomo de un hidrógeno. Tomado, sin embargo, en conjunción con los resultados de Lenard para la absorción de los rayos catódicos, estos números parecen favorecer la hipótesis de que los portadores de las cargas son más pequeños que los átomos de hidrógeno".

Los resultados de Philipp Lenard a los que se refería Thomson indicaban que si los rayos catódicos eran partículas cargadas moviéndose a grandes velocidades, entonces el tamaño de los portadores debían ser pequeños comparados con las dimensiones de átomos o moléculas ordinarias ${ }^{46}$.

Es, sin embargo, el artículo del Philosophical Magazine el que se considera habitualmente como la verdadera "puesta de largo" del descubrimiento de los corpúsculos a los que pronto todos llamarían electrones ${ }^{47}$. 


\section{J.J. Thomson y la génesis del descubrimiento del electrón}

Figura 2

TH E

I,ONDON, EDINBURGH, AND DUBLIN

PHILOSOPHICAL MAGAZINE

$\triangle N D$

JOURNAL OF SCIENCE.

[FIFTH SERIES.]

OCTOBER 1897.

XL. Cathoile Kays. By J. J. Tномвом, M.A., F.R.S., Cavendish Professor of Experimentul Physics, Cumbridge*.

7 HE experiments $\dagger$ discussed in this paper were undertiken 1 in the hope of gaining sume information ns to the nuture of the Cathode Rays. The most diverse opinions are hold as to these rays; according to the almost unanimous opinion of German physicists they are due to some process in the ather to which-inasmuch as in a uniform magnetic field their course is circular and not rectilinear-no phenoInenon hitherto observed is analogous : another view of these rays is that, so far from being wholly æotherial, they are in fact wholly material, and that they murk the paths of particlos of matter charged with negative electricity. It would seem at first sight that it ought not to be difficult to discriminate betwoen views so different, yet experience shows that this is not the case, as annongst the physicists who have most deeply studied the subject can be found supporters of either theory.

The electrified-particle theory has fur purposes of rescarch a great advantage over the ætherial theory, since it is definite and its consequences can be predicted; with the ætherial theory it is impossible to predict what will happen under any given circumstances, as on this theory we are dealing with hitherto

- Communicated by the Author.

+ Sume of these experiments have already been described in a paper read before the Cambridge Philusuphical Socinty (Proceedings, vol. ix. 1897) and in a Fridny Evening Discourse at the Royal Institution ('Electrician,' Mny 21, 1897).

Plit. Mfag. S. 5. Vol. 44. No. 269. Oct. 1897.

Y

Primera página del artículo de J. J. Thomson de 1897 en el que introdujo los "corpúsculos" que luego todos denominarían *electrones*. 
Comienza este justamente famoso trabajo con una frase modesta: "Los experimentos considerados en este artículo fueron emprendidos con la esperanza de obtener alguna información sobre la naturaleza de los rayos catódicos".

En el primer experimento tratado, Thomson seguía el camino que recorrían los rayos catódicos mediante el procedimento de reconocer la fosforencencia que emitían cuando golpeaban las paredes de cristal del tubo. Desviando los rayos a través de una pequeña hendidura en un electrodo conectado a un electrómetro, JJ demostraba que la «electrificación negativa sigue la misma trayectoria que los rayos, y que esta electrificación negativa está indisolublemente conectada con los rayos catódicos".

El segundo estadio consistía en utilizar un tubo de cristal casi idéntico a los que se emplean en la actualidad en los osciloscopios y aparatos de televisión. Aplicando una diferencia de potencial a dos placas paralelas, a través de las cuales pasaban los rayos catódicos, se producía una desviación de éstos, con la que comprobaba que se comportaban como era de esperar que lo hicieran partículas cargadas en campos eléctricos y magnéticos. Es entonces cuando se preguntaba (p. 302): «¿Qué son estas partículas? ¿son átomos, o moléculas, o materia en una forma todavía más fina de división?» Y añadía: "Para arrojar alguna luz sobre este punto, he realizado una serie de medidas del cociente entre la masa de estas partículas y la carga que transportan".

Pasaba entonces Thomson a describir varios experimentos que le permitían calcular $e / \mathrm{m}$. El primero de estos experimentos, por citar uno de ellos, se basaba en desviaciones producidas por un campo magnético e implicaba mediciones, por separado, de la cantidad de electricidad transportada por las supuestas partículas de los rayos catódicos, su energía cinética y el radio de curvatura de sus trayectorias. Utilizando como gases a través de los cuales pasaban los rayos, aire, hidrógeno y ácido carbónico, Thomson obtenía como valores para $\mathrm{e} / \mathrm{m}$ :

$$
0,4 \times 10^{-7}, 0,5 \times 10^{-7} \text { y } 0,9 \times 10^{-7} \text {, }
$$

respectivamente, en unidades C.G.S. electrostáticas.

Con tales datos, Thomson podía avanzar hacia conclusiones más fundamentales: "De estas determinaciones" -escribía en la página 310 - «vemos que el valor de $\mathrm{e} / \mathrm{m}$ es independiente de la naturaleza del gas, y que su valor $10^{-7}$ es muy pequeño comparado con $10^{-4}$, que es el valor de esta cantidad más pequeño previamente conocido, y que es el valor para el ión de hidrógeno en la electrolisis». Y repetía lo que ya había dicho en la Royal Institution: "La pequeñez de $\mathrm{e} / \mathrm{m}$ 


\section{J.J. Thomson y la génesis del descubrimiento del electrón}

Figuras 3 y 4
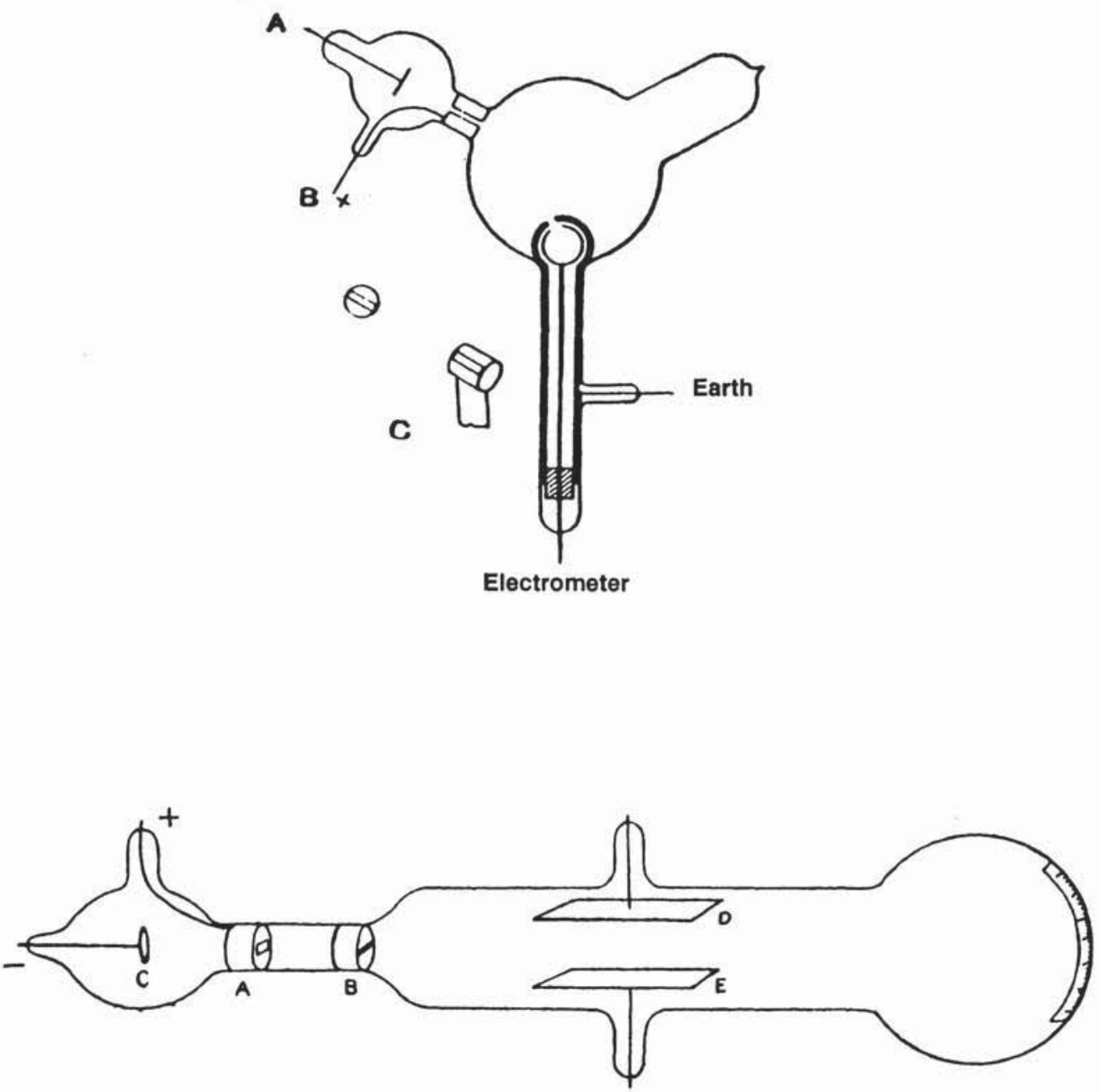

Figuras incluidas en el artículo de 1897, que representan los instrumentos utilizados por Thomson en sus experimentos. 
puede ser debida a que $m$ sea pequeño o a que $e$ sea grande, o a una combinación de ambos. Que los portadores de las cargas en los rayos catódicos son pequeños comparados con las moléculas ordinarias viene demostrado, creo yo, por los resultados de Lenard de que la luminosidad de la fosforescencia producida por estos rayos disminuye con la longitud del camino recorrido por el rayo». Estaba claro, por consiguiente que los «dos puntos fundamentales acerca de estos portadores... son: (1) que estos portadores son los mismos cualquiera que sea el gas a través del cual pasa la descarga, (2) que el recorrido libre medio no depende nada más que de la densidad del medio atravesado por estos rayos".

En cuanto a la manera que el director del Cavendish favorecía para entender los resultados que había obtenido, era la siguiente ( $p$. 311):

"La explicación que me parece describe de la manera más simple y directa los hechos se basa en el punto de vista de la constitución de los elementos químicos que ha sido sostenida favorablemente por muchos químicos: este punto de vista es que los átomos de elementos químicos distintos son agregaciones diferentes de átomos del mismo tipo. En la forma en que esta hipótesis fue enunciada por Prout, los átomos de los diferentes elementos eran átomos de hidrógeno; la hipótesis no se sostiene en esta precisa forma, pero si sustituimos el hidrógeno por alguna sustancia primordial desconocida, $\mathrm{X}$, entonces no hay nada que sea inconsistente con esta hipótesis, que ha sido apoyada recientemente por Sir Norman Lockyer por razones que se derivan del estudio de los espectros estelares.

$\mathrm{Si}$, en el muy intenso campo eléctrico de las proximidades del cátodo, las moléculas del gas se disocian y rompen, no en átomos químicos ordinarios, sino en estos átomos primordiales, que llamaremos por brevedad corpúsculos, y si estos corpúsculos están cargados con electricidad y son proyectados del cátodo por el campo eléctrico, se comportarán exactamente igual que los rayos catódicos".

Éste es, para mi propósito, el punto esencial. Los "corpúsculos», futuros electrones, entraban en escena. Thomson había formulado con claridad una hipótesis revolucionaria: que los átomos tradicionales eran divisibles en $\longrightarrow$ que contenían-corpúsculos.

Un detalle interesante surge cuando se constata que Thomson no hacía referencia alguna en su artículo a los trabajos de Stoney. Muy posiblemente esto se debiese a que no deseaba especular acerca de la posible relación entre las cargas que él había descubierto y las 


\section{J.J. Thomson y la génesis del descubrimiento del electrón}

unidades de carga introducidas previamente por Stoney (y por otros, como ya vimos) a propósito de la electrolisis. Seguramente por eso mismo recurrió a un nombre diferente, "corpúsculo" y no "electrón». No estaba en condiciones de saber si se trataba de los mismos entes físicos. Si hubiese utilizado el valor de la unidad de carga eléctrica que se derivaba de los procesos electrolíticos, entonces habría llegado inmediatamente a que sus corpúsculos debían tener una masa 1.000 veces menor que la del hidrógeno.

Aunque las consecuencias de su artículo de 1897 eran claras, hubo que esperar a 1899 para que Thomson completase realmente su camino al descubrimiento del electrón. Lo hizo en un nuevo artículo en Philosophical Magazine: "On the mases of the ions in gases at low pressures" ${ }^{48}$. Para no alargar ya más mi discusión, me limitaré a reproducir extractos de la introducción (pp. 547-548), así como la conclusión final:

«En un artículo anterior (Phil. Mag. Oct. 1897), di una determinación del valor del cociente entre la masa, $m$, del ión y su carga, e, en el caso de la corriente de electrificación negativa que constituye los rayos catódicos. Los resultados de esta determinación... demuestran que el valor de este cociente es mucho menor que el cociente correspondiente en el caso de la electrolisis de soluciones de ácidos y sales, y que es independiente del gas a través del cual pasa la descarga y de la naturaleza de los electrodos. En estos experimentos solamente se determina el valor de $e / m$, y no los valores por separado de $e$ y de $m \ldots$ El presente artículo contiene la descripción de medidas para $e / m$ y para $e$ para la electrificación negativa producida por un filamento de carbono incandescente en una atmósfera de hidrógeno".

En cuanto a la conclusión que extraía, no podía ser más clara (p. 563): "Los experimentos que acabo de describir, demuestran, tomados en conjunción con los previos sobre el valor de $\mathrm{e} / \mathrm{m}$ para los rayos catódicos (J. J. Thomson, Phil. Mag. Oct. 1897), que en gases a bajas presiones la electrificación negativa... está formada por unidades, cada una de las cuales posee una carga de electricidad fija; la magnitud de esta carga negativa es de aproximadamente $6 \times 10^{-10}$ unidades electrostáticas, y es igual a la carga positiva transportada por el átomo de hidrógeno en la electrolisis de soluciones".

Los fenómenos electrolíticos y los observados en tubos de rayos catódicos se encontraban finalmente, y así no es demasiado sorprendente que a la postre el, más atractivo, nombre "electrón" acabara imponiéndose. En el camino, y además de los "corpúsculos" thomsonianos, 
se quedaron expresiones como: "iones univalentes" (Helmholtz), "monada elemental" (S. P. Thompson), «electrión" (Kelvin) ${ }^{49}$, o "sub-átomos".

Para concluir esta sección y para que nos hagamos idea de la novedad que significaba el "penetrar" realmente en la estructura atómica, voy a citar a continuación unos párrafos de la conferencia que William Crookes pronunció en su calidad de presidente de la reunión de la British Association for the Advancement of Science que tuvo lugar en Bristol en $1898^{50}$ :

\begin{abstract}
«Hace varios años me ocupé de la constitución de la materia en lo que me atreví a denominar el cuarto estado. Intenté penetrar en el tormentoso misterio del átomo. ¿Qué es un átomo? ¿Es un átomo simple un espacio sólido, líquido o gaseoso? Cada uno de estos estados involucra ideas que pueden pertenecer sólamente a vastas colecciones de átomos. $\mathrm{Si}$, al igual que Newton intentamos visualizar un átomo como un cuerpo duro, esférico, o, con Boscovitch y Faraday, lo consideramos como un centro de fuerza, o aceptamos la teoría del átomo vorticial de Lord Kelvin, un átomo aislado es una entidad desconocida difícil de concebir. Las propiedades de la materia - ś́lida, líquida, gaseosa- son debidas a moléculas en un estado de movimiento. Por consiguiente, la materia tal y como la conocemos implica esencialmente un modo de movimiento; y el propio átomo -intangible, invisible e inconcebible- es su base material, y puede, de hecho, ser considerado la única materia verdadera. El espacio implicado en los movimientos de los átomos no tiene más razón de ser denominado materia que si denominásemos a la esfera de influencia de un cuerpo de artilleros - estando la esfera ocupada por los misiles de plomo en vuelo- plomon.
\end{abstract}

\title{
11. La recepción del descubrimiento de Thomson
}

Estudiar la recepción dada a un descubrimiento científico es una tarea compleja, en tanto que tal recepción se manifiesta de muy diversas maneras. Una forma, por ejemplo, de apreciar la recepción del trabajo de Thomson sobre el electrón es a través de las nominaciones que JJ recibió para el premio Nobel de física. Afortunadamente, desde hace algún tiempo se dispone de información sobre este punto ${ }^{51}$.

La primera vez que Thomson fue nominado para el premio Nobel de física fue en 1902, el año en que se otorgó por segunda vez (en aquella ocasión fueron Hendrik Antoon Lorentz y Pieter Zeeman los galardonados; en 1901 lo había sido Wilhelm Röntgen). Sorprendentemente, fue el astrónomo estadounidense, George Ellery Hale, quien 
nominó a Thomson. En 1903, sin embargo, no recibió ninguna mención (aquel año fueron Henri Becquerel, Marie y Pierre Curie los afortunados); por contra, él sí fue uno de los nominadores, recayendo su voto en John William Strutt (Rayleigh; volvio a votarlo en 1904, año en que Rayleigh recibio el premio). En 1904 volvio a recibir una nominación (de Nikolai Umov).

No fue, sin embargo, hasta 1905 cuando el nombre de JJ Thomson adquirió verdadera fuerza como candidato al Nobel. Aquel año (en el que el galardonado fue Philipp Lenard) fue nominado por diez científicos: Marie y Pierre Curie, Joseph Norman Lockyer, Oliver Lodge, John Lubbock, William Ramsay, Rayleigh, Silvanus P. Thompson, William Thomson (esto es, lord Kelvin) y William Henry White. Finalmente, su turno llegó en 1906, aunque entonces recibio menos nominaciones que el año anterior: ocho (de Adolf von Baeyer, Christian Christiansen, Lodge, Lubbock, Ramsay, Rayleigh, Silvanus Thompson y Emil Warburg).

Si buscamos en las conferencias pronunciadas en las reuniones de la British Association for the Advancement of Science, nos encontramos con que inicialmente el eco de los trabajos de Thomson no fue excesivo. Así, es significativo señalar que en su conferencia - ya citada- presidencial de la reunión de 1898 de la Association, Crookes, que sí se detuvo a comentar desarrollos recientes en el campo del electromagnetismo (las relaciones entre materia y éter, entre otras) y de radiaciones como los rayos X, no se ocupase de los trabajos de JJ Thomson sobre el electrón, a los que, sin embargo, se refería indirectamente de la manera siguiente ${ }^{62}$ : "Mi idea de un cuarto estadio de la materia propuesta en 1881 [nota bene: Phil. Trans. Parte 2, 1881, pp. 433-4], y a la que al principio se opusieron tanto en el extranjero como en casa, está siendo aceptada ahora. Es apoyada por el profesor J. J. Thomson [nota bene: Phil. Mag., Octubre 1897, p. 312]" "53. Por el contrario, las aportaciones de H. A. Lorentz, J. Larmor, G. F. FitzGerald o J. Stoney merecían más atención que las de JJ. Tampoco encontramos en la conferencia presidencial de la Sección A (pronunciada aquel año por W. E. Ayrton) referencia alguna a los trabajos de Thomson (ni en los restantes trabajos presentados).

Comentando en 1900 el libro de Joseph Larmor, Aether and Matter, que acaba de aparecer, George Francis FitzGerald escribía, ignorando a Thomson ${ }^{54}$ : «Desde que Faraday descubriera la ley de la electrolisis, según la cual la ruptura de un enlace químico allí corresponde a la transferencia de la misma cantidad de electricidad, independientemente de qué clase de enlace sea, ha estado en el aire científico que la 
electricidad al igual que la materia puede ser atómica. En este contexto, Maxwell se vio conducido a utilizar el término "átomo de electricidad", aunque lo minusvaloró como bastante contrario a todo el espíritu de su tratado; pero ahora se reconoce ampliamente, gracias a Mr. Larmor y al profesor Lorentz de Leyden, que muy lejos de que esta idea sea contraria al espíritu del tratado de Maxwell es en realidad muy compatible con él, y para algunos puntos de vista es incluso esencial. El libro de Mr. Larmor está dedicado en gran medida a elaborar este punto de vista de la naturaleza de la electricidad. Supone que estos átomos de electricidad, o electrones como los denomina $\mathrm{Mr}$. Stoney, producen el fenómeno de corrientes eléctricas al moverse, y o bien se mueven de molécula a molécula de materia, como en la conducción metálica ordinaria, o por átomos de materia, como en la conducción electrolítica".

No me resulta fácil comprender esta aparente resistencia por parte de algunos científicos británicos a reconocer el mérito de Thomson, resistencia que se ponía de manifiesto en ocasiones haciendo que JJ compartiese protagonismo con otros físicos, Stoney, Lorentz y Larmor, en particular. Tal vez - sería necesario explorar con más detalle esta posibilidad - la explicación se encuentre en el apego que aquellos científicos sentían por el concepto de éter ${ }^{55}$, y en el hecho de que en los trabajos de Thomson - quien, por otra parte, también sentía una gran inclinación por tal concepto- los corpúsculos eléctricos (o electrones) aparecían bajo un ropaje - el del análisis puramente experimentalen buena medida $\longrightarrow$ en principio - ajeno a la física del éter, lo que ciertamente no ocurría en las presentaciones de Larmor y Lorentz.

Algo de esta posibilidad se trasluce en la presentación - divulgación realmente_ que otro británico, William Cecil Dampier Whetham, realizó en 1904 de los últimos desarrollos llevados a cabo en la física, y en la que se pueden leer párrafos como los siguientes ${ }^{56}$ :

"Grande ha sido la revelación que sigue. Los átomos aislados de electricidad negativa - los electrones, como han sido denominados por Stoney- han sido identificados por los trabajos de Thomson, Lorentz y Larmor, con la base física de la materia, con los corpúsculos, o subátomos, de los cuales, combinados en números variables y en disposiciones diferentes, están constituidos los átomos químicos, durante mucho tiempo considerados como las últimas unidades indivisibles".

Con este desarrollo, señalaba Whetham (p. 8), «el sueño de los antiguos filósofos de una base común para la materia se ha realizado mediante 


\section{J.J. Thomson y la génesis del descubrimiento del electrón}

el análisis experimental». Pero lo más interesante para las posibilidades que estoy considerando aquí son párrafos como el siguiente ${ }^{57}$ :

«El corpúsculo de J. J. Thomson, el electrón de Stoney, Larmor y Lorentz, es representado en el mundo etéreo por la concepción de Larmor de un centro de tensión intrínseca. Al contrario que el átomo vorticial, este centro de tensión no es parte del medio ya que está separado del resto para siempre; sólo persiste la tensión, la parte del éter que es afectada por ella cambia constantemente según se mueve el subátomo. El éter está inmovil, y el robusto fantasma que constituye la materia flota hacia arriba y hacia abajo a través de él según pasan las ondas sobre la superficie del mar. Tal persistencia temporal con movilidad espacial sería imposible para una forma de tensión en cualquier éter sólido elástico, pero puede producirse en un éter rotacional del tipo descrito por Lord Kelvin".

Muy diferente es el caso del significado que la nueva partícula de Thomson tenía para un, también reciente, campo de investigación que se abría camino con fuerza creciente: el de la radiactividad.

\section{La radiactividad y el electrón}

Pronto se hizo evidente que los corpúsculos/electrones desempeñaban un papel importante para entender los procesos que tenían lugar en los procesos radiactivos. Una forma de comprobar esta afirmación es observar qué dijeron al respecto los líderes en aquel entonces nuevo dominio de la física: el de la radiactividad.

En el caso de Marie Curie, que, como se indicó, nominó (al igual que su esposo, Pierre) a Thomson para el premio Nobel de Física en 1905 , si buscamos entre sus publicaciones nos encontramos con que la primera vez que mencionó explícitamente a JJ Thomson y al electrón fue en un artículo de carácter general que publicó en 1900: «Les nouvelles substances radioactives» ${ }^{58}$. Es extremadamente interesante citar la mención más extensa que se hace de ambos en aquel trabajo (nótese que todavía no se hace uso del nombre «electrón»):

"Hasta el momento, siempre se ha encontrado que allá donde hay electricidad hay materia; los rayos catodicos son cargas de electricidad, y ello lleva a pensar que son materiales. En la teoría de Crookes, los rayos catódicos son una proyección de la materia electrizada que procede del cátodo; es la "materia radiante" de Crookes. La experiencia ha mostrado que la velocidad de los rayos catódicos es muy grande, apenas 
tres veces menor que la de la luz. J. J. Thomson ha demostrado que si se considera que los rayos catódicos son partículas materiales electrizadas, estas partículas transportan un peso 1.000 veces menor que el de un átomo de hidrógeno. No serían ya por tanto los átomos libres de la química, sino sub-átomos todavía mucho más pequeños, y animados de velocidades prodigiosas. Al igual que en un tubo de vacío estas partículas se escapan del cátodo, el radio las enviaría al espacio de forma continua. La materia radiactiva sería, por consiguiente, materia en donde reina un estado de movimiento interior violento, materia a punto de romperse. Si es así, el radio debe perder constantemente peso. Pero la pequeñez de las partículas es tal que aunque sea fácil de constatar la carga eléctrica enviada al espacio, la masa correspondiente debe ser absolutamente insignificante; calculando se encuentra que harían falta millones de años para que el radio perdiera un equivalente en miligramos de su peso. Es imposible de llevar a cabo la verificación.

La teoría materialista de la radiactividad es muy seductora. Explica bien los fenómenos de la radiactividad. Sin embargo, adoptando esta teoría es preciso concluir que la materia radiactiva no se encuentra en un estado químico ordinario; los átomos no están ahí en un estado estable, porque se radian partículas más pequeñas que el átomo. El átomo, indivisible desde el punto de vista químico, es aquí divisible, y los sub-átomos están en movimiento. La materia radiactiva experimenta por consiguiente una transformación química que es la fuente de la energía radiada; pero no se trata de una transformación química ordinaria, porque éstas dejan al átomo invariable. En la materia radiactiva, si hay algo que se modifica, es a la fuerza el átomo, porque es al átomo al que se asocia la radiactividad".

Sorprendentemente, Ernest Rutherford, alumno y colaborador de Thomson durante un tiempo, fue mucho más lento que Marie Curie en reconocer a través de la letra impresa la importancia del trabajo de su antiguo mentor (tal vez porque no encontró ocasión de hacerlo antes). La primera referencia a los resultados de JJ que he localizado en un artículo de Rutherford data de 1902, en un trabajo titulado "The existence of bodies smaller than atoms" ${ }^{59}$. Al principio, no obstante, la mención a Thomson no dejaba de contener un punto de duda ${ }^{60}$ : «J. J. Thomson también encontró que el cociente $\mathrm{e} / \mathrm{m}$ era independiente del gas en el tubo de vacío, demostrando que, posiblemente, de diferentes tipos de materia se producían partículas del mismo tamaño. Es, sin embargo, posible explicar este resultado suponiendo que la descarga es transportada en todos los casos por el resto de vapor de agua que está siempre presente en el tubo de vacío". A continuación las dudas comenzaban a desaparecer y Rutherford afirmaba: «Se había dado de 
esta manera una confirmación completa a la teoría de proyección de los rayos catódicos, y la importancia del trabajo fue reconocida inmediatamente por físicos continentales». De hecho, en el resto del artículo continuaba comentando favorablemente la demostración de una unidad de carga elemental, especialmente habida cuenta de que los distintos métodos empleados (por Thomson y otros) conducían a resultados idénticos. Así, próximo al final de su artículo, manifestaba ${ }^{61}$ : "La existencia física de los electrones es aceptada ahora por muchos científicos y hay un gran número de prominentes fĩsicos que están desarrollando matemáticamente la secuencia lógica de la idea. Necesito solamente mencionar unos pocos de entre los más destacados: Drude, Voigt, Riecke, en Alemania, Lorentz y Zeeman, en Holanda, Poincaré y Becquerel, en Francia, J. J. Thomson, Schuster, Lodge y Lord Kelvin, en Inglaterra, para demostrar que la idea tiene una base de apoyo sólida entre los físicos más capaces».

El electrón, vemos, se había establecido ya firmemente en el cada vez más variado y complejo universo de la física. Aunque acompañado por muchas otras partículas «elementales», continúa disfrutando de semejante privilegiada posición hasta la fecha.

\section{Notas}

1 El nombre de Mathematical Tripos tiene que ver con el hecho de que al principio el examen se realizaba en una habitación en la que el estudiante se sentaba en una banqueta de tres patas (tripode). La denominación comenzó a ser utilizada a partir de 1824. Estrictamente el nombre oficial era: "Senate House Examination".

2 Paul Forman, John L. Heilbron y Spencer Weart, Physics circa 1900. Personnel, funding, and productivity of the academic establishments, Historical Studies in the Physical Sciences 5 (1975), pp. 32-33. Ver, asimismo, P. M. HARMAN, "Introduction", Wranglers and Physicists. Studies on Cambridge Mathematical Physics in the Nineteenth Century, P. M. HARMAN, ed. (Manchester University Press, Manchester 1985), pp. $1-11$.

3 Ver especialmente: J. LARMOR, Aether and Matter (Cambridge University Press, Cambridge 1900).

4 J. J. Thomson, Recollections and Reflections (G. Bell and Sons, Londres 1936), p. 63.

5 Macmillan, Cambridge 1859. Esta obra se reproduce también en The Scientific Papers of James Clerk Maxwell, W. D. NIVEN, ed. (Dover, Nueva York 1965), vol. I, pp. 288-376, y en Maxwell on Saturn's Rings, Stephen G. BRUSH, C. W. EveriTT y Elizabeth GaRBER, eds. (The MIT Press, Cambridge, Mass. 1983), pp. 69-158.

6 No pasó mucho tampoco hasta que fue elegido assistant lecturer en Matemáticas del Trinity. Sus obligaciones en este puesto le obligaban a dedicar dieciocho horas semanales a dar clases. 
7 Philosophical Magazine 5, 229-234 (1881).

8 A. SCHUSTER, The Progress of Physics during 33 Years (1875-1908) (Cambridge University Press, Cambridge 1911), p. 50. En esta obra se tratan también algunos de los puntos que estoy mencionando.

9 Esta ley (de Faraday) básica de la electrolisis se puede enunciar también de la manera siguiente: «La separación química de materia que tiene lugar en la electrolisis es proporcional a la cantidad de electricidad que pasan, o: «la acción química es exactamente proporcional a la cantidad de electricidad que pasa a través de una solución que sufre una descomposición (electrolítica)». Entre los trabajos de Faraday sobre la electrolisis, hay que destacar: "Experimental researches in electricity.- Fifth series. On electro-chemical decomposition", Philosophical Transactions of the Royal Society $123,675-710$ (1833), y «Experimental researches in electricity.- Seventh series. On electro-chemical decomposition, continued. On the absolute quantity of electricity associated with the particles or atoms of matter", Philosophical Transactions of the Royal Society 124, 77-122 (1834).

10 L. Pearce Williams, Michael Faraday (Chapman and Hall, Londres 1965). La cita siguiente, que procede de la "Seventh series" (ver nota precedente), se reproduce en la p. 257 del libro de Williams.

11 J. Clerk MaXwell, A Treatise on Electricity and Magnetism (Dover, Nueva York 1954), vol. I, p. 374. Aquellos interesados en las ideas de Maxwell sobre los puntos que estoy comentando deben consultar también su Theory of Heat (Longmans, Green and Co., Londres 1872), en particular las páginas 305-306 «Molecular theory of electrolysis*) y 306-308 ( Molecular theory of radiation*).

12 Ibid., p. 380.

13 Hermann von Helmholtz, *The modern development of Faraday's conception of electricity*, Journal of the Chemical Society (London) 39, 277-304 (1881); reimpreso en Selected Writings of Hermann von Helmholtz, Russell KAHL, ed. (Wesleyan University Press, Middletown 1971), pp. 409-436; p. 415. Un artículo reciente en el que se analiza este trabajo de Hemholtz dentro del contexto de su obra en física y química es el de Helge KRAGH, «Between physics and chemistry. Helmholtz's route to a theory of chemical thermodynamics", en Hermann von Helmholtz and the Foundations of Nineteenth-century Science, David CaHAN, ed. (University of California Press, 1993), pp. 403-431.

14 Ibid., p. 422

15 Ibid., p. 423.

$16 \mathrm{H}$. EBERT, «Heat of dissociation according to the electrochemical theory», Philosophical Magazine 38, 332-336 (1894); p. 332.

17 Sobre Stoney y sus trabajos acerca del electrón, véase J. G. O’HARA, «George Johnstone Stoney, F.R.S., and the concept of the electron", Notes and Records of the Royal Society of London 29, 265-276 (1975). Aunque este artículo cubre un espectro más amplio que el que yo trato aquí, creo que algunos aspectos que se abordan en el presente artículo (como las muestras que ofrezco de las opiniones de Stoney) son más completos que los de O'Hara.

18 Philosophical Magazine 38, 418-420 (1894).

19 G. J. STONEY, "On the physical units of nature", Philosophical Magazine 11, 381-390 (1881). Notese que la conferencia de Helmholtz fue en abril de 1881, y la de Stoney en Belfast, en febrero del mismo año.

20 F. RichaRz, "On Helmholtz's electrochemical theory", Philosophical Magazine 39, 529-531 (1895). 


\section{J.J. Thomson y la génesis del descubrimiento del electrón}

21 G. J. STONEY, «On the physical units of nature», p. 383.

22 J. G. STONEY, «On the cause of double lines in spectra*, Report of the Sixty-First Meeting of the Association for the Advancement of Science held at Cardiff in August 1891 (John Murray, Londres 1892), pp. 574-575; p. 574. No es ésta, sin embargo, la referencia más citada para la introducción por parte de Stoney del nombre *electrón». La que se ofrece en la mayoría de los artículos que se refieren a esta aportación de Stoney (ver, por ejemplo, los comentarios de E. A. DAVIS, en Science in the Making [Taylor and Francis, Londres 1997], vol. 2, p. 314) es: Philosophical Transactions of the Royal Society 4, 518 (1891). Desgraciadamente, en el momento de tener que entregar este artículo no he podido todavía acceder a este trabajo, a pesar de los esfuerzos realizados. Y no estoy seguro de que tal referencia sea la correcta, ya que no aparece en ese número de la revista en la colección existente en la Academia de Ciencias de Madrid. A la vista de cómo finalizó Stoney su intervención en Cardiff («Una descripción completa de la investigación está siendo impresa por la Royal Dublin Society en sus Scientific Transactions»), es muy probable que la referencia exacta sea Transactions of the Royal Dublin Society (acaso 4, 563-608 [1891], uno de sus artículos).

23 Ver, Elisabeth CRAWFond, Arrhenius. From Ionic Theory to the Greenhouse Effect (Science History Publications, Canton, Mass. 1996).

24 Sobre el Laboratorio Cavendish, consultar: A History of the Cavendish Laboratory, 1871-1910 (Longmans, Green, and Co., Londres 1910), y J. G. CROWTHER, The Cavendish Laboratory, 1874-1974 (Science History Publications, Nueva York 1974).

25 William Thomson había sido la primera elección para ocupar la cátedra, pero éste no aceptó abandonar su puesto en Glasgow; después se intentó una aproximación a Hermann von Helmholtz, que también resultó infructuosa; fue entonces cuando se eligi6 a Maxwell.

26 Recollections and Reflections, p. 95.

27 The Electrical Researches of the Honourable Henry Cavendish, F.R.S. Written between 1771 and 1781, Edited from the Original Manuscripts on the Possession of the Duke of Devonshire, K.G. by J. Clerk Maxwell, F.R.S. (Cambridge University Press, Cambridge 1879).

28 Sobre la elección de Rayleigh, consultar Robert John STRUTT, Life of John William Strutt, Third Baron Rayleigh (The University of Wisconsin Press, Madison 1968), cap. VII («The Cambridge professorship at the Cavendish laboratory»).

29 «Hacia el final de mi estancia en Owens, Arthur Schuster también estaba trabajando en el laboratorio y dio un curso al que asistí sobre el Treatise on Electricity and Magnetism de Maxwell, que acababa de ser publicado"; Recollections and Reflections, p. 22. Aunque no abordo este tema aquí, Schuster también efectuó investigaciones que le aproximaron al descubrimiento del electrón. Véase en este sentido, Stuart M. FEFFER, "Arthur Schuster, J. J. Thomson, and the discovery of the electron", Historical Studies in the Physical and Biological Sciences 20, 33-61 (1989).

30 E. A. Davis e I. J. FAlConer, J. J. Thomson and the Discovery of the Electron (Taylor and Francis, Londres 1997), pp. 45-46. En lo que sigue, yo corrijo, sin embargo, algunas de las razones ofrecidas por estos autores.

31 En su ya mencionado libro - The Progress of Physics - Schuster citaba (pp. 51-52) alguna posibilidad para mantener la idea de la corriente eléctrica como un fenómeno asociado con el éter.

32 W. CROOKES, "Experimental contributions to the theory of the radiometer", Proceedings of the Royal Society 25, 304-314 (1876-1877). 
33 Una visión general de los trabajos de Crookes es la dada por W. H. BROcK, "Crookes, William*, Dictionary of Scientific Biography, Charles Coulson GillespIE, ed. (Charles Scribner, Nueva York s.f.), pp. 474-482; p. 477. Ver también Per F. DAHL, Flash of the Cathode Rays (Institute of Physics Publishing, Bristol 1997), p. 64 y ss.

$34 \mathrm{~W}$. CROOKES, *On the illumination of lines of molecular pressure, and the trajectory of molecules", Philosophical Transactions of the Royal Society 170, 135-164 (1879); 135. Las «líneas de presión molecular* de Crookes estaban, sin duda, inspiradas en las líneas de fuerza/campo electromagnéticas de Faraday.

35 Sobre las ideas de Hertz, y otros como Lenard, véase Per F. DAHL, Flash of the Cathode Rays.

36 J. PERRIN, «Nouvelles propriétés des rayons cathodiques», Comptes Rendus de l'Académie des Sciences 121, 1130 (1895); reproducido en Oeuvres Scientifiques de Jean Perrin (Centre National de la Recherche Scientifique, París 1950), pp. 3-5.

37 The Correspondence between Sir George Gabriel Stokes and Sir William Thomson, Baron Kelvin of Largs, vol. 2 (1870-1901), David B. WilsoN, ed. (Cambridge University Press, Cambridge 1990), p. 635.

38 Se debe referir a $\mathrm{Ph}$. LENARD, „Über Kathodenstrahlen in Gasen von atmosphärischem Druck und im äussersten Vakuum* Sitzungsberichte der Berliner Akademie der Wissenschaften, y Annalen der Physik und Chemie 51, 225-267 (1894) y “Über die magnetische Ablenkung der Kathodenstrahlen*, ibid. 52, 23-33 (1894). Para un comentario del primer trabajo, véase P. F. DAHL, Flash of the Cathode Rays, p. 85 y ss.

39 The Correspondence between Sir George Gabriel Stokes and Sir William Thomson, Baron Kelvin of Largs, p. 636.

40 Kelvin, "Presidential address", Proceedings of the Royal Society 54, 377-389 (1894), 56, 522, 546, 649.

41 J. J. Thomson, «Presidential address*, Report of the Sixty-sixth Meeting of the Association for the Advancement of Science held at Liverpool in September 1896 (John Murray, Londres 1896), pp. 699-706; pp. 701-702.

42 Para tal explicación, ver, en el aspecto histórico, E. A. DAviS e I. J. FALConer, J. J. Thomson and the Discovery of the Electron. Muy recomendable es la reconstruccion que Steven WeINBERG realizó de los razonamientos teóricos utilizados por Thomson: Particulas subatómicas (Prensa Científica, Barcelona 1985). En esta referencia no sólo se comparan los valores obtenidos por Thomson con los actuales, sino que también se critican algunos de los pasos y resultados de JJ.

43 J. J. THOMSON, «Cathode rays", The Electrician 39, 104 (1897), también publicada en Proceedings of the Royal Institution (30 de abril de 1897), pp. 1-14.

44 J. J. Thomson, "Cathode rays", Proceedings of the Royal Institution, p. 8.

45 Ibid., p. 14.

46 Ya me he referido a los trabajos de Lenard; ver nota 38.

47 J. J. Thомson, «Cathode rays», Philosophical Magazine 44, 293-316 (1897).

48 Philosophical Magazine 48, 547-567 (1899).

49 Silvanus P. THOMPSON, The Life of William Thomson, Baron Kelvin of Largs (Macmillan, Londres 1910), vol. 2, p. 1074.

50 W. CROOKES, "Presidential address", Report of the Sixty-Eight Meeting of the British Association for the Advancement of Science held at Bristol in September 1898 (John Murray, Londres 1899), pp. 3-38; p. 20. 


\section{J.J. Thomson y la génesis del descubrimiento del electrón}

51 Elisabeth Crawford, J. L. HeIlbron y Rebecca Ullrich, The Nobel Population, 1901-1937. A Census of the Nominators and Nominees for the Prizes in Physics and Chemistry (Office for History of Science and Technology, University of California, Berkeley/ Office for History of Science, Uppsala University, Uppsala 1987).

52 W. CROOKES, «Presidential address», p. 24.

53 También, en otro lugar de su conferencia, señalaba (p. 23): «Puede recordarse que J. J. Thomson ha encontrado para los rayos catódicos una velocidad de más de 10.000 kilómetros por segundo».

54 G. F. FitzGerald, "Aether and matter", Electrician, julio de 1900; reproducido en The Scientific Writings of the Late George Francis FitzGerald, J. LARMOR, ed. (Hodges, Figgis and Co., Dublin 1902), pp. 511-515; 512.

55 Stanley GolberG, «In defense of ether: the British response to Einstein's theory of relativity", Historical Studies in the Physical Sciences 2, 89-124 (1970). Ver, asimismo, el análisis, en varios aspectos alternativos al de Goldberg, de José M. SÁNCHEz RoN, "The reception of special relativity in Great Britain", en The Comparative Reception of Relativity, Thomas F. GLICK, ed. (Reidel, Dordrecht 1987), pp. 27-58.

56 W. C. DAmpier Whetham, The Recent Development of Physical Science (John Murray, Londres 1904), p. 3.

57 Ibid., pp. 279-280.

58 Revue des Sciences (Revue Rose) 14, 65 (1900); reimpreso en Oeuvres de Marie Sklodowska Curie, Iréne Joliot CuRIE, ed. (Pnstwowe Wydawnictwo Naukowe, Varsovia 1954), pp. 95-105. El nombre de Thomson aparece en las páginas 97 y 104; la cita que sigue procede de las páginas 104-105.

59 Transactions of the Royal Society of Canada 8, 79-86 (1902); reimpreso en The Collected Papers of Lord Rutherford of Nelson, James CHADwick, ed. (George Allen and Unwin, Londres 1962), vol. I, pp. 403-409.

60 Ibid., The Collected Papers of Lord Rutherford of Nelson, p. 406.

61 Ibid., p. 409. 